\title{
Image-Guided Hyaluronic Acid Injection and Knee Bracing Significantly Improve Clinical Outcomes for High-Grade Osteoarthritis
}

\author{
Terry K. Morgan ${ }^{1 *}$, Emilie Jensen ${ }^{1}$, Jeong Lim² ${ }^{2}$ and Russell Riggs ${ }^{3}$
}

\begin{abstract}
Background: Intra-articular hyaluronic acid (HA) injection is an intermediate option between analgesics and knee joint replacement in patients with osteoarthritis (OA). Our objective was to test whether image-guided HA injections may improve knee OA outcomes after 6 months of treatment independent of potential covariates.

Methods: This is a retrospective case series with multivariate outcome-based analysis of 207 consecutive adult patients with mild to severe knee OA treated at a single out-patient clinic employing fluoroscopy-guided HA injections. We employed a customized pain (scored 0-10) and function (scored 0-120) questionnaire based on the Likert scale to compare baseline scores with 6-month outcomes. Linear and logistic (based on >9-point score improvement) regression analysis was used to adjust for potential covariates, including grade of disease, patient age, gender, body mass index, smoking history, medical history (e.g., diabetes or heart disease), use of daily pain medications, fish oil supplementation, knee bracing, and physical therapy.
\end{abstract}

Results: Significant covariates included OA grade, knee bracing, and analgesic use. Most of the study subjects were women (124/207, $60 \%$ ) and obese (113/207, 55 \%). Clinically significant improvements in index scores (>9 points) at 6 months were observed in more than $50 \%$ of cases post-image-guided HA injection. Regression analysis revealed a complimentary affect with knee bracing, especially in severe grade 4 disease (odds ratio 5.5 [1.14-27.0], $P<0.05)$. Daily analgesic use reflected a poor clinical response to treatment.

Conclusions: Our data suggest image-guided HA injections coupled with knee bracing may benefit patients with moderate to severe knee osteoarthritis.

Key Points:

- Image-guided hyaluronic acid injections significantly improve clinical outcomes at 6 months for mild, moderate, and severe knee osteoarthritis.

- Knee bracing is a significant covariate for clinical improvement in severe grade 4 disease.

- Daily analgesic use is associated with high-grade disease and less clinical improvement.

Keywords: Knee osteoarthritis; Hyaluronic acid; Intra-articular injection; Fluoroscopy

\footnotetext{
* Correspondence: morgante@ohsu.edu

'Department of Pathology, Oregon Health \& Science University, 3181 SW

Sam Jackson, Mail Code L-471, Portland, OR 97239, USA

Full list of author information is available at the end of the article
}

\section{实 Springer}

(c) 2015 Morgan et al. Open Access This article is distributed under the terms of the Creative Commons Attribution 4.0 International License (http://creativecommons.org/licenses/by/4.0/), which permits unrestricted use, distribution, and reproduction in any medium, provided you give appropriate credit to the original author(s) and the source, provide a link to the Creative Commons license, and indicate if changes were made. 


\section{Background}

Osteoarthritis (OA) of the knee is a leading cause of adult disability [1], affecting approximately $10-15 \%$ of Americans over the age of 60 years [2]. The estimated cost to the United States economy is over $\$ 100$ billion per year [3, 4], and the prevalence of the disease is expected to increase over the next decade due to obesity and aging of the population.

A wide variety of treatment options are available [5], including weight loss, knee bracing, physical therapy, analgesics, intra-articular injections (e.g., hyaluronic acid [HA], methylprednisolone), and surgery (e.g., debridement or joint replacement). Non-surgical conservative approaches such as HA injection provide an intermediate approach between analgesics and joint replacement with the objective of decreasing pain, improving function, and delaying the need for joint replacement. Recent meta-analyses provide conflicting opinions about the efficacy of HA injections [6-8], which may affect providers' willingness to recommend this type of treatment. However, we suspect many patients may potentially benefit from image-guided injections $[9,10]$ after controlling for covariates.

We hypothesized that image-guided intra-articular HA injections improve 6-month clinical outcomes in adults with mild to severe knee OA. Moreover, supplemental knee bracing and analgesic support may improve outcomes compared with image-guided HA injection alone.

\section{Methods}

\section{Study Design}

This is a retrospective case series with multivariate outcome-based analysis using an Oregon Health \& Science University Institutional Review Board approved protocol to extract clinical chart data from 207 adult patients (25-105 years old) with mild (grade 2), moderate (grade 3), or severe (grade 4) knee OA treated at a single out-patient clinic in 2013. All study subjects were Caucasian with 6 months of clinical follow-up after a series of three fluoroscopy-guided $(1.0 \mathrm{ml}$ of IsoVue contrast injected into the knee with a 21-gauge needle) HA (Euflexxa, 1 \% Sodium Hyaluronate, Ferring Pharm. Inc. Parsippany, NJ) injections administered 1 week apart by an experienced physician (RR). Clinical data were extracted from uniform patient questionnaires using a customized pain (scored 0-10) and function (scored 0-120) assessment based on the Likert scale $[7,11]$. Physical examination and clinical history were screened for potential covariates, including the grade of osteoarthritis, which was defined by the KellgrenLawrence criteria [12]: grade 2 (mild) had definite joint space narrowing and osteophytic lipping, grade 3 (moderate) also showed sclerosis and possible bone contour deformity, and grade 4 (severe) had severe sclerosis, joint narrowing, large osteophytes, and bone contour deformities. Patient age, gender, body mass index, smoking history, significant medical history (e.g., diabetes or heart disease), subjective reporting of over-the-counter daily analgesics (e.g., NSAIDs), daily fish oil supplements, and duration of disease were also documented. More than half of the study subjects (110/207) were fitted with a noncustomized single-hinged medial off-loading knee brace (V/Q OrthoCare, Vista, CA) after HA injection for the duration of the 6-month interval available for outcome metrics. Knee bracing was recommended to patients with limitations in activity defined by the ability to ambulate up and down a flight of stairs \pm knee brace. Physical therapy was recommended for all study subjects. Of these, 100 study subjects regularly participated in knee-centric physical therapy (8-10 sessions over 6-month study period). Patients completed follow-up questionnaires and had a physical examination at 2 and 6 months post-imageguided HA injection. This archived patient chart data was extracted into a coded file without patient identifiers (e.g., name, birthdate) for statistical analysis. Extraction quality assurance was verified by $10 \%$ independent review (20/207 cases).

\section{Statistical Analyses}

All statistical analyses were performed using SAS (version 9.4, SAS Institute, Cary, NC). Patient demographics, potential covariates, and outcome metrics were examined by univariate analysis using the Student $t$-test. Potential covariates (e.g., knee bracing, analgesics) were analyzed as binary variables (yes or no). In the cases with bilateral knee involvement, the highest OA grade knee was employed for analysis. The primary outcome metric was pain and function outcomes at 2 and 6 months compared with baseline (before first HA injection) for linear regression analysis, and "clinically significant" improvement was defined as $>9$-point decrease in score for logistic regression analysis. Subjective pain index (0-10) was a secondary outcome analyzed by linear regression.

\section{Results}

Multivariate regression analysis demonstrated that knee osteoarthritis grade was the most significant covariate affecting clinical outcomes at 2 months $(P<0.0001)$ and 6 months $(P<0.0001)$ post-image-guided HA injection. Therefore, all analyses were reported separately for each OA grade: mild (grade 2), moderate (grade 3), and severe (grade 4) disease (Table 1). As expected, univariate analysis showed that increasing severity of knee OA was related to increasing subject age and body mass index. Most of the study subjects were obese with BMIs $\geq 30.0$ (113/207, $55 \%$ ) and most were women (124/207, $60 \%$ ). Univariate analysis did not show a relationship between OA grade and smoking, daily analgesic use, or physical 
Table 1 Patient demographics and outcomes treated by image-guided hyaluronic acid injection

\begin{tabular}{|c|c|c|c|c|}
\hline & OA grade $2(n=58)$ & OA grade $3(n=98)$ & OA grade $4(n=51)$ & $P$ value $X^{2}$ or ANOVA \\
\hline Age (mean, SD) & $56.3 \pm 12.4$ & $64.0 \pm 11.3$ & $69.8 \pm 12.2$ & $P<0.0001$ \\
\hline Male gender $(n,[\%])$ & $20(35 \%)$ & $41(42 \%)$ & $21(42 \%)$ & $P=0.62$ \\
\hline Body mass index (mean, SD) & $29.6 \pm 6.5$ & $31.2 \pm 6.5$ & $34.6 \pm 9.3$ & $P<0.01$ \\
\hline Never smoked $(n,[\%])$ & $49(85 \%)$ & $86(88 \%)$ & $42(82 \%)$ & $P=0.29$ \\
\hline Physical therapy (n, [\%]) & $27(47 \%)$ & $44(45 \%)$ & $20(39 \%)$ & $P=0.72$ \\
\hline Daily analgesics $(n,[\%])$ & $23(40 \%)$ & $41(42 \%)$ & $26(51 \%)$ & $P=0.39$ \\
\hline Knee bracing $(n,[\%])$ & $20(35 \%)$ & $60(61 \%)$ & $31(61 \%)$ & $P<0.01$ \\
\hline Fish oil $(n,[\%])$ & $43(74 \%)$ & $64(65 \%)$ & $43(84 \%)$ & $P=0.05$ \\
\hline Function index baseline (mean, SD) & $34.7 \pm 16.1$ & $46.0 \pm 15.4$ & $48.1 \pm 13.3$ & $P<0.0001$ \\
\hline Index at 6 months (mean, SD) & $27.2 \pm 15.9$ & $26.3 \pm 14.9$ & $36.4 \pm 17.7$ & $P<0.01$ \\
\hline Mean improvement $( \pm \mathrm{SD})$ & $6.7 \pm 19.0$ & $19.3 \pm 16.8$ & $12.5 \pm 14.6$ & $P<0.001$ \\
\hline Percentage improvement $( \pm$ SD) & $19 \% \pm 32 \%$ & $40 \% \pm 32 \%$ & $26 \% \pm 31 \%$ & $P<0.001$ \\
\hline >9-point improvement ( $n,[\%])$ & $32(55 \%)$ & $67(68 \%)$ & $26(51 \%)$ & $P=0.07$ \\
\hline Average pain baseline (mean, SD) & $5.5 \pm 2.5$ & $6.2 \pm 2.1$ & $7.1 \pm 2.1$ & $P<0.01$ \\
\hline Pain at 6 months (mean, SD) & $3.9 \pm 2.0$ & $3.8 \pm 2.1$ & $5.2 \pm 2.5$ & $P<0.01$ \\
\hline Mean improvement (mean, SD) & $1.66 \pm 2.1$ & $2.74 \pm 2.5$ & $2.3 \pm 2.8$ & $P=0.05$ \\
\hline Percentage improvement $( \pm$ SD) & $21 \% \pm 38 \%$ & $40 \% \pm 37 \%$ & $25 \% \pm 47 \%$ & $P=0.04$ \\
\hline
\end{tabular}

Univariate analysis of patient demographics and treatment outcomes relative to knee osteoarthritis (OA) severity (grades 2-4). Data are presented as the mean \pm standard deviation (SD) or percent (\%) within OA grade

therapy. Moderate to severe OA were more likely to be fitted with a knee brace. Surprisingly, the greatest frequency of improvement was seen in more severe disease (68 \% of study subjects), which also showed the most improvement in pain index (40\% compared with $21 \%$ in mild disease).

Regression analysis revealed that knee bracing provided a positive complimentary effect to HA injection in severe OA cases (Table 2) to improve outcomes with an odds ratio of $5.5(1.14-27.0)$ and $P$ value $<0.05$. Analgesic use reflected a poor clinical response to treatment, rather than positive additive effect as first postulated.
Physical therapy significantly decreased subjective pain index for mild OA $(P<0.01)$, but it had no effect on moderate or severe disease. Outcomes at 2 months were not significantly different than 6-month outcomes in this case series (data not shown) with the exception that bracing appeared to positively affect outcomes in moderate cases at 2 months (odds ratio 2.17 [0.86-5.48], $P$ value 0.02 ), which remained a positive effect at 6 months, but the effect was not statistically significant (Table 2). Mild OA did not respond to knee bracing at either 2 or 6 months of evaluation, although logistic regression suggested a positive trend $(P=0.19)$.

Table 2 Regression analysis of significant covariates for clinical improvement

\begin{tabular}{|c|c|c|c|c|c|c|}
\hline & OA grade $2(n=58)$ & & OA grade $3(n=98)$ & & OA grade $4(n=51)$ & \\
\hline Function index linear regression & Parameter estimate & $P$ value & Parameter estimate & $P$ value & Parameter estimate & $P$ value \\
\hline Knee bracing & +2.69 & 0.67 & +2.56 & 0.51 & +10.81 & 0.04 \\
\hline Daily analgesics & -5.58 & 0.38 & -8.6 & 0.03 & -13.94 & 0.003 \\
\hline Logistic regression (>9-point improvement) & Odds ratio [95 \% C.I.] & $P$ value & Odds ratio [95 \% C.I.] & $P$ value & Odds ratio [95 \% C.I.] & $P$ value \\
\hline Knee bracing & $2.4[0.66-8.81]$ & 0.19 & $1.22[0.45-2.22]$ & 0.70 & $5.54[1.14-27.0]$ & 0.03 \\
\hline Daily analgesics & $0.12[0.03-0.53]$ & 0.005 & $0.55[0.18-1.63]$ & 0.28 & $0.12[0.03-0.61]$ & 0.009 \\
\hline Average pain index linear regression & Parameter estimate & $P$ value & Parameter estimate & $P$ value & Parameter estimate & $P$ value \\
\hline Knee bracing & +0.94 & 0.25 & +0.30 & 0.67 & +1.81 & 0.10 \\
\hline Daily analgesics & -0.59 & 0.46 & -1.06 & 0.14 & -2.15 & 0.03 \\
\hline Physical therapy & +2.11 & 0.004 & -0.25 & 0.73 & -1.30 & 0.22 \\
\hline
\end{tabular}

In addition to intra-articular HA injection, knee bracing significantly improved clinical outcomes with positive (+) effect on clinical indices at 6 months in severe (grade 4) osteoarthritis (OA) analyzed by both linear and logistic regression. In contrast, daily analgesic use was a sign of poor clinical response to treatment with worsening scores (-) 


\section{Discussion}

Intra-articular HA knee injections are commonly performed by orthopedic surgeons [13] and increasingly by primary care physicians [5]. Although recent metaanalyses provide conflicting opinions about the efficacy of HA injections [6-8], it should be no surprise that image-guided injections may yield significantly better results $[9,10]$. Our retrospective analysis of fluoroscopyguided HA knee injections supports this conclusion. Moreover, our multivariate regression analysis reveals OA grade and bracing may significantly affect clinical outcomes.

Most of the image-guidance literature centers on corticosteroid injections. For example, Jones et al. [10] reported that ultrasound guidance yielded clinically significant improvement in 28/54 (52\%) of their study subjects, while only $7 / 30(23 \%)$ showed improvement without imaging. They attributed this discrepancy to incorrectly placed injections, which missed the joint space. Indeed, meta-analyses have shown that image-guided intra-articular knee injections yield $~ 97 \%$ accuracy for needle placement compared with $\sim 81 \%$ accuracy without imaging [9], which of course means better efficacy and increased cost-effectiveness [14, 15].

An important limitation of our retrospective case series is the fact that all the study subjects received image-guided injections. They were not randomized into guided and non-guided HA injections for comparison. However, our data suggest future studies should test whether guidance together with knee bracing improves short-term and long-term outcomes when treating knee OA with HA. This is especially important for severe disease, which is a group most likely to require joint replacement and may benefit most from combined HA injection and knee bracing.

Analgesic use showed no positive effects in our data, and the literature suggests they have little clinical effect [16]. Notably, daily analgesic use was not prescribed in our study. Patients who reported daily analgesic use were more likely to have high-grade disease, less likely to show a functional improvement by 6 months, and less likely to report a reduction in the subjective pain index relative to baseline. We therefore suspect these patients may be more likely to be long-term non-responders to HA injection and knee bracing. This data also suggest the mechanism of action of HA injections may not be solely anti-inflammatory.

The biological actions of HA viscosupplementation are only beginning to be understood. For example, HA may increase synovial fluid viscosity [17] and regulate a number of cytokines involved in both anti-inflammatory activity and extracellular matrix restorative mechanisms. Recent studies exploring the effects of HA on activated CD4 positive $T$ cells in synovial fluid [18] and reduction of joint tissue catabolism [19] are intriguing.
Both mechanisms suggest that in some patients HA may have more long-term positive effects than only short-term improvements in pain and patient function. Long-term outcome data were not available for our analysis, but they are needed to test whether image-guided HA injections with knee bracing may delay joint replacement. Over $75 \%$ of OA patients with joint space narrowing progress to total knee replacement within 18 years of diagnosis [20]. Delaying joint replacement is therefore very important because the lifespan of the device is $10-15$ years. In an obese aging population, cost-effective interventions like HA injection and knee bracing make sense.

\section{Conclusions}

Our data suggest image-guided HA injections coupled with knee bracing may benefit patients with moderate to severe knee osteoarthritis. With the current rates of obesity and the advanced aging of the population, it is reasonable to speculate that many more patients will require revision surgery if alternative intervention methods like viscosupplementation are not effective. A randomized prospective trial to thoroughly evaluate the short-term and long-term impact of image-guided HA injection with and without knee bracing is needed.

\section{Abbreviations \\ BMl: body mass index; HA: Hyaluronic acid; OA: Osteoarthritis.}

\section{Competing interests}

Dr. Russell Riggs is the owner and medical director of the Reflex Clinic. Although he participated in the original documentation of patient data during treatment, these clinical charts were not intended for publication. Dr. Riggs did not participate in retrospective data extraction from the Reflex Clinic clinical records or data analysis performed at Oregon Health \& Science University. However, this study was funded by the Reflex Clinic to support independent coded data extraction by the Morgan laboratory and statistical analysis.

Emilie Jensen and Drs. Morgan and Lim declare they have no competing interests.

\section{Authors' contributions}

TKM was principal investigator and managed all aspects of study design, data collection, and analysis. EJ was a summer intern at the Morgan laboratory and collected coded data from the Reflex Clinic archives for analysis. $J$ analyzed the data. RR provided access to his clinic's data, necessary funding, and the clinical methods described in the manuscript. All authors participated in the drafting of this manuscript and have given final approval for publication.

\section{Author details}

${ }^{1}$ Department of Pathology, Oregon Health \& Science University, 3181 SW Sam Jackson, Mail Code L-471, Portland, OR 97239, USA. ${ }^{2}$ Department of Public Health \& Preventative Medicine, Oregon Health \& Science University, Portland, OR, USA. ${ }^{3}$ Reflex Clinic, Portland, OR, USA.

Received: 19 May 2015 Accepted: 25 August 2015

Published online: 15 September 2015

\section{References}

1. Peat G, McCarney R, Croft P. Knee pain and osteoarthritis in older adults: a review of community burden and current use of primary health care. Ann Rheum Dis. 2001;60(2):91-7. 
2. Dillon C, Rasch E, Gu Q, Hirsch R. Prevalence of knee osteoarthritis in the United States: arthritis data from the Third National Health and Nutrition Examination Survey 1991-94. J Rheumatol. 2006;33(11):2271-9.

3. Buckwalter J, Saltzman C, Brown T. The impact of osteoarthritis: implications for research. Clin Orthop Relat Res. 2004;427(Suppl):S6-15.

4. Jackson D, Simon T, Aberman H. Symptomatic articular cartilage degeneration: the impact in the new millennium. Clin Orthop Relat Res. 2001;391(Suppl):S14-25.

5. Crawford D, Miller L, Block J. Conservative management of symptomatic knee osteoarthritis: a flawed strategy? Ortho Rev (Pavia). 2013;5(1), e2.

6. Bannuru R, Natov N, Dasi U, Schmid C, McAlindon T. Therapeutic trajectory following intra-articular hyaluronic acid injection in knee osteoarthritis - meta-analysis. Osteoarthr Cartil. 2011;19(6):611-9.

7. Leighton R, Akermark C, Therrien R, Richardson J, Andersson M, Todman M et al. NASHA hyaluronic acid vs methylprednisone for knee osteoarthritis: a prospective, multicenter, randomized, non-inferiority trial. Osteoarthr Cartil. 2014;22:17-25.

8. Rutjes A, Juni P, da Costa B, Trelle S, Nuesch E, Reichenbach S Viscosupplementation for osteoarthritis of the knee. Ann Intern Med. 2012;157(3):180-91.

9. Berkoff D, Miller L, Block J. Clinical utility of ultrasound-guidance for intra-articular knee injections: a review. Clin Interv Aging. 2012;7:89-95.

10. Jones A, Regan M, Ledingham J, Pattrick M, Manhire A, Doherty M Importance of placement of intra-articular steroid injections. BMJ. 1993;307(6915):1329-30.

11. Bellamy N, Buchanan W, Goldsmith C, Campbell J, Stitt L. Validation study of WOMAC: a health status instrument for measuring clinically important patient relevant outcomes to antirheumatic drug therapy in patients with osteoarthritis of the hip or knee. J Rheumatol. 1988;15(12):1833-40.

12. Kellgren J, Lawrence J. Radiographic assessment of osteoarthritis. Ann Rheum Dis. 1957:16(4):494-502.

13. Yiasemidou M, Munir U, Glassman D, Teanby D. Efficacy and safety of biweekly viscosupplementation regimen for knee osteoarthritis. J Knee Surg. 2015. doi:10.1055/s-0034-1394300

14. Hatoum H, Fierlinger A, Lin S, Altman R. Cost-effectiveness analysis of intra-articular injections of a high molecular weight bioengineered hyaluronic acid for the treatment of osteoarthritis knee pain. J Med Econ. 2014;17(5):326-37.

15. Sibbitt W, Brand P, Kettwich L, Chavez-Zhiang N, Delea S, Bankhurst A. A randomized controlled trial evaluating the cost-effectiveness of sonographic guidance for intra-articular injection of the osteoarthritic knee. J Clin Rheumatol. 2011;17(8):409-15.

16. Scott $D$, Berry $H$, Capell $H$. The long-term effects of non-steroidal anti-inflammatory drugs in osteoarthritis of the knee: a randomized placebo-controlled trial. Rheumatology (Oxford). 2000;39(10):1095-101.

17. Zhou PH, Liu SQ, Peng H. The effect of hyaluronic acid on IL-1 beta-induced chondrocyte apoptosis in a rat model of osteoarthritis. J Orthop Res. 2008:26(12):1643-8

18. Lurati A, Laria A, Mazzocchi D, Re K, Marrazza M, Scarpellini M. Effects of hyaluronic acid (HA) viscosupplementation on peripheral Th cells in knee and hip osteoarthritis. Osteoarthr Cartil. 2015;23(1):88-93.

19. Sundman E, Cole B, Karas V, Valle C, Tetreault M, Mohammed H, et al. The anti-inflammatory and matrix restorative mechanisms of platelet-rich plasma in osteoarthritis. Am J Sports Med. 2014;42(1):35-41.

20. Wolfe F, Lane $N$. The long term outcome of osteoarthritis: rates and predictors of joint space narrowing in symptomatic patients with knee osteoarthritis. J Rheumatol. 2002:29(1):139-46.

\section{Submit your manuscript to a SpringerOpen ${ }^{\circ}$ journal and benefit from:}

- Convenient online submission

- Rigorous peer review

- Immediate publication on acceptance

- Open access: articles freely available online

- High visibility within the field

- Retaining the copyright to your article

Submit your next manuscript at $\gg$ springeropen.com 